\title{
Postnatal Neurogenesis in the Kitten Retina
}

\author{
PAMELA RAYMOND JOHNS,' ANNE C. RUSOFF ${ }^{2}$ AND MARK WM. DUBIN ${ }^{3}$ \\ ${ }^{1}$ Department of Anatomy, Medical Science Building II; ${ }^{2}$ Division of Biological Sciences, \\ Natural Sciences Building, University of Michigan, Ann Arbor, Michigan 48109; and \\ ${ }^{3} \mathrm{MCDB}$, University of Colorado, Box 347 , Boulder, Colorado 80309
}

\begin{abstract}
Postnatal neurogenesis in the kitten retina was studied using ${ }^{3} \mathrm{H}$-thymidine radioautography. Kittens were injected with ${ }^{3} \mathrm{H}$-thymidine at 1 day, 10 days, 3 weeks or 4 weeks after birth and allowed to survive until 14 weeks of age. Labeled neuronal nuclei were not found in the ganglion cell layer in any of the retinas, but they were seen in the other nuclear layers of the same retinas. In retinas from kittens injected at one day after birth, the peripheral $80 \%$ of the length of the retina (in sections cut parallel to the dorsoventral meridian) contained labeled nuclei; the central $20 \%$, around the optic disc, contained no labeled nuclei. Near the ora serrata most nuclei in both inner and outer nuclear layers were labeled. Away from the ora serrata the proportion of labeled to unlabeled nuclei gradually decreased. Labeled nuclei extended farther centrally in the inner than the outer nuclear layer. The same pattern of labeling was repeated in retinas from kittens injected at ten days after birth, but fewer nuclei were labeled, and the central, unlabeled region around the optic disc was longer $-55 \%$ of the length of the retina. Only a few nuclei near the ora serrata were labeled in retinas from kittens injected at three weeks after birth, and no labeled neurons were found in kittens injected at four weeks.

From these results we conclude that all of the ganglion cells in the kitten retina are present by one day after birth, as are all of the other neurons in the central retina. In peripheral regions of the inner and outer nuclear layers, proliferation of cells destined to become neurons continues up to three weeks after birth.
\end{abstract}

The pattern and temporal sequence of retinal development is remarkably similar in all vertebrates (Ramón y Cajal, '60). During the early stages, germinal cells proliferate throughout the presumptive retinal epithelium, and mitotic figures are seen all along the ventricular surface (Fujita, '62). Neuronal differentiation starts in the central region of the retina when the first ganglion cells withdraw from the mitotic cycle (Hinds and Hinds, '74). While the central retina is maturing, mitotic activity continues in peripheral regions, and a central-to-peripheral gradient of maturation exists for the remainder of the developmental period (Sidman, '60). In some mammals, such as mice and rats, the later stages of retinal neurogenesis occur in the first weeks after birth (Sidman, '60; Denham, '67).
Retinal neurogenesis has not been thoroughly studied in the cat, although it is known that at birth, the kitten's retina is not fully differentiated. Mitotic figures are present (Donovan, '66), implying that the retina does not have its full complement of cells at birth. However, quantitative studies of the number and kinds of retinal cells produced postnatally have not been done.

To define the pattern and time course of postnatal neurogenesis we have used ${ }^{3} \mathrm{H}$-thymidine radioautography. This study shows that retinal neurogenesis continues up to three weeks after birth in the kitten and that the neurons produced postnatally are in the inner and outer nuclear layers. All of the retinal ganglion cells are formed earlier, before postnatal day 1.

Reprint requests should be made to Mark Wm. Dubin- 


\section{METHODS}

Kittens, 1 day, 10 days, 3 weeks or 4 weeks old, were given a single intraocular injection of either 50 or $100 \mu \mathrm{Ci}$ of ${ }^{3} \mathrm{H}$-thymidine (New England Nuclear, specific activity 40-60 Ci/ mmole) in $50 \mu \mathrm{l}$. The animals were anesthetized with fluothane (Halothane), and the isotope was injected with a needle inserted into the vitreous humor through a hole in the dorsal-temporal region of the pars plana. All animals survived to 14 weeks of age. The eyes were fixed in Bouin's solution, embedded in paraffin or carbowax, sectioned meridionally (transversely) at $10 \mu \mathrm{m}$, parallel to the dorsoventral plane, and processed for radioautography (Kopriwa and Leblond, '62). Slides were dipped in NTB-2 emulsion (Eastman-Kodak, Rochester, New York), diluted 1:1 with water; after an exposure time of two to four weeks, the radioautographs were developed and stained through the emulsion with hematoxylin and eosin.

Cells with labeled nuclei were recognized by the presence of silver grains in the overlying emulsion. The linear extent of retinal regions containing labeled nuclei was measured in five sections each from eyes injected at 1 day, 10 days or 3 weeks after birth. Separate measurements were made of labeled regions in the inner and outer nuclear layers on both the dorsal and the ventral sides of the disc. To ensure that comparable areas were selected from each retina, all measured sections passed through the optic disc; the area centralis was not included in these sections.

The large number of labeled nuclei in retinas from animals injected at 1 or 10 days of age made the labeled regions easy to see at low power with a stereomicroscope fitted with a darkfield base (Wild). The boundaries between labeled and unlabeled regions of both inner and outer nuclear layers were marked in ink on the coverslip. The radioautographs from the eyes of kittens injected at three or four weeks of age had many fewer labeled nuclei, so the labeled regions were not as easily seen; the boundaries were marked after inspection with a compound microscope. The radioautographs were then traced using a camera lucida at a total magnification of $45 \times$, and the positions of the ink marks and the optic disc were noted. The length of the retina, defined as the linear distance from one retinal margin (ora serrata) to the other, was determined by laying a non-elastic string along the retinal tracings and then measuring the length of string with a ruler. The data presented are measurements from one eye at each age; radioautographs from additional eyes showed similar results.

Sections from the eye of a newborn kitten, prepared for another light microscopic study (M. A. Ransford and M. W. Dubin, in preparation), were used to determine the position of mitotic figures in the retina at birth. Five sections, cut parallel to the dorsoventral plane in the same manner as the radioautographs, were selected; again all of the sections contained the optic disc. The segments containing mitotic figures, both dorsal and ventral to the optic disc, were marked in ink on the coverslip. The sections were traced and lengths measured, as described above for the radioautographs.

\section{RESULTS}

The retina of a newborn kitten is not fully differentiated. Although the ganglion cell and inner plexiform layers can be recognized across the entire extent of the retina, the more scleral layers - the inner nuclear layer, the outer plexiform layer and the outer nuclear layer - are well-differentiated only in central regions. In the peripheral retina, the outer plexiform layer is barely discernible as a thin zone, free of nuclei. Cells on the vitreal side of the outer plexiform layer, in the presumptive inner nuclear layer, appear undifferentiated, with elongated nuclei containing pale, homogenous chromatin. On the scleral side of the outer plexiform layer, in the presumptive outer nuclear layer, the nuclei appear more differentiated, with the dark, clumped chromatin characteristic of photoreceptors in the mature retina (fig. 1). These observations agree in general with those of Donovan ('66), Cragg ('75), Morrison ('77), Tucker ('78), and Vogel ('78).

The total retinal length in the newborn eye we examined was $19.1 \mathrm{~mm}$. Mitotic figures, seen at the retina-pigmented epithelium boundary (fig. 1), were found in the dorsal retina near the ora serrata and up to $9.8 \mathrm{~mm}$ centrally (range of measurements on 5 sections: 8.7-10.4 mm). On the ventral side, mitotic fig. ures were found up to $5.9 \mathrm{~mm}$ (range: $5.0-6.8$ $\mathrm{mm}$ ) away from the ora serrata. The zone of mitotic activity thus encompassed the peripheral $80 \%$ of the length of the retina, in the dorsoventral plane represented in these sections.

Labeled nuclei were seen in radioautographs of retinas from animals injected one day to three weeks after birth and allowed to 

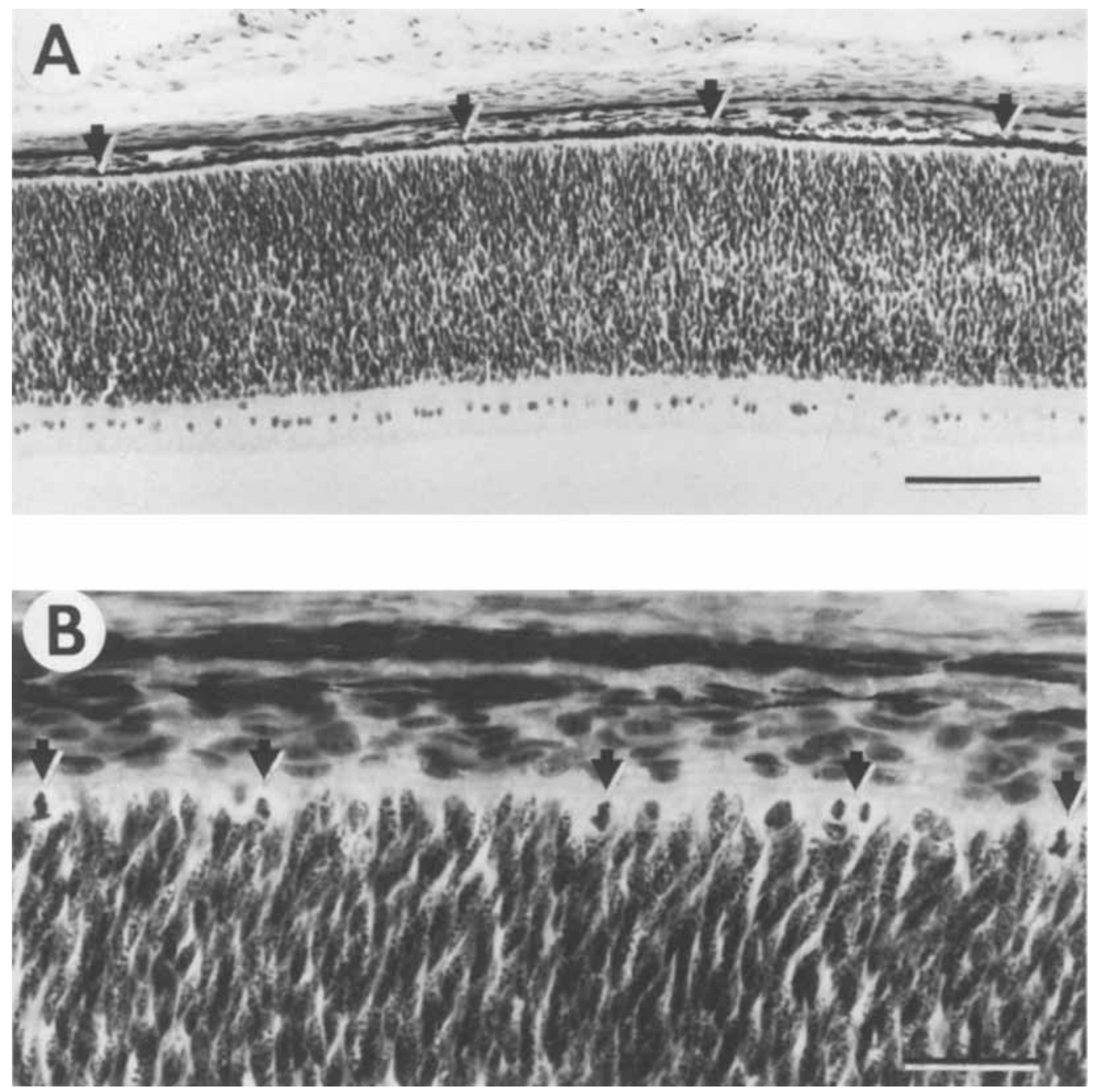

Fig. 1 Photomicrographs of the peripheral retina from a newborn kitten.

A The ganglion cell layer is discrete, but the more scleral layers have not yet differentiated. The arrows point to mitotic figures located at the boundary between neural retina and pigmented epithelium. The scale equals $100 \mathrm{mi}$ crometers.

B A higher magnification view of the mitotic figures in a similar field. The scale equals 25 micrometers.

survive until 14 weeks of age. The presence of silver grains (label) over a nucleus indicates that the cell incorporated ${ }^{3} \mathrm{H}$-thymidine and ceased dividing shortly thereafter; when it differentiated, its nucleus retained the label. These labeled nuclei were distributed unequally among the retinal layers. Particularly striking was the absence of labeled ganglion cell nuclei in all of the radioautographs (fig. 2 ). This means that the ganglion cells com- plete their terminal mitotic division before the kitten is 24 hours old. (Injections of thy. midine were not attempted during the first postnatal day because of the precarious physiological state of newborn kittens.)

Following injection at one day after birth and survival to 14 weeks of age, labeled nuclei were found in the layers scleral to the ganglion cell layer along much of the length of the retina (fig. 3). The distribution of labeled nu- 


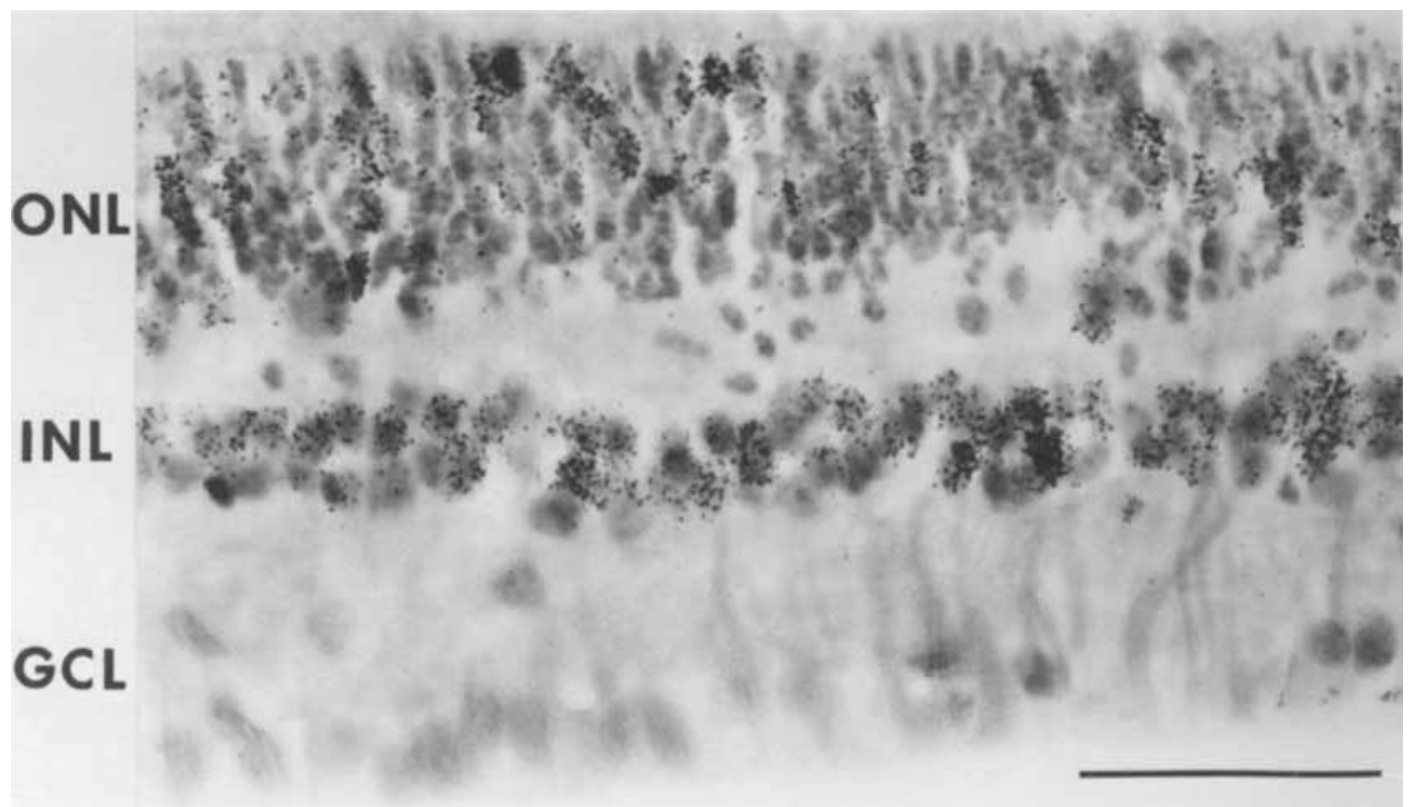

Fig. 2 Radioautograph of the retina from a 14-week-old kitten, injected with ${ }^{3}$ H-thymidine on the first day after birth. Labeled nuclei are seen in the outer nuclear layer (ONL) and in the inner nuclear layer (INL). No nuclei are labeled in the ganglion cell layer (GCL). The scale equals 50 micrometers.

clei varied both along and across retinal laminae (fig. 3). The lengths of the retinal segments, on the dorsal and ventral sides of the optic disc, which contained labeled nuclei are given in table 1.

In the inner nuclear layer, labeled nuclei were found up to 10-11 mm away from the ora serrata (table 1). Since the entire length of this retina was $25.9 \mathrm{~mm}$ (table 1), the fraction of inner nuclear layer with labeled nuclei was about $80 \%$. Within this region not every nucleus was labeled, and the proportion of labeled to unlabeled nuclei (labeling index) varied with retinal position: most nuclei near the ora serrata were labeled (figs. 4A,B), but fewer were labeled more centrally (fig. 3 : arrow 2). Close to the central boundary of the labeled segment only scattered labeled nuclei were seen (fig. 3: arrow 3). Since the retinas were embedded in wax and thick $(10 \mu \mathrm{m}) \mathrm{sec}$ tions were used for the radioautographs, the various cell types in the inner nuclear layer could not be distinguished. Thus, we do not know what kinds of cells were labeled in this layer.

In the outer nuclear layer of the same retinas (injected 1 day after birth), the labeled region was shorter, extending only about $8 \mathrm{~mm}$ centrally from the ora serrata, or $65 \%$ of the total length of the retina. As in the inner nuclear layer, the labeling index decreased from the ora serrata toward the center of the retina. At each point along the retina the labeling index for the outer nuclear layer appeared less than that for the adjacent region of the inner nuclear layer. For example, in figure 2 approximately one half of the nuclei in the inner nuclear layer are labeled compared with only about one fourth of those in the outer nuclear layer. (These proportions were obtained by counting nuclei on a camera lucida drawing made with a $50 \times$ oil immersion objective; a nucleus was considered labeled if overlain by five or more silver grains.)

Fig. 3 Dark-field mosaic of a radioautograph from a 14-week-old kitten, injected with ${ }^{3} \mathrm{H}$-thymidine on the first day after birth. Only dorsal retina is shown. The layers containing labeled nuclei can be identified in the inset (at a higher magnification). The silver grains appear as bright dots; the pigmented layers (PL) scleral to the retina also shine brightly, but they are not labeled. Other abbreviations in the inset are the same as in figure 2. Arrow 1 points to the ora serrata; central to arrow 1, many nuclei are labeled, especially in the inner nuclear layer (INL). Arrow 2 points to the boundary between regions containing high and low proportions of labeled nuclei. Arrow 3 points to the most central labeled nucleus in the INL. Arrow 4 points to the optic disc. The asterisk indicates a distortion in the outer nuclear layer (see text). The scale equals $1 \mathrm{~mm}$. 


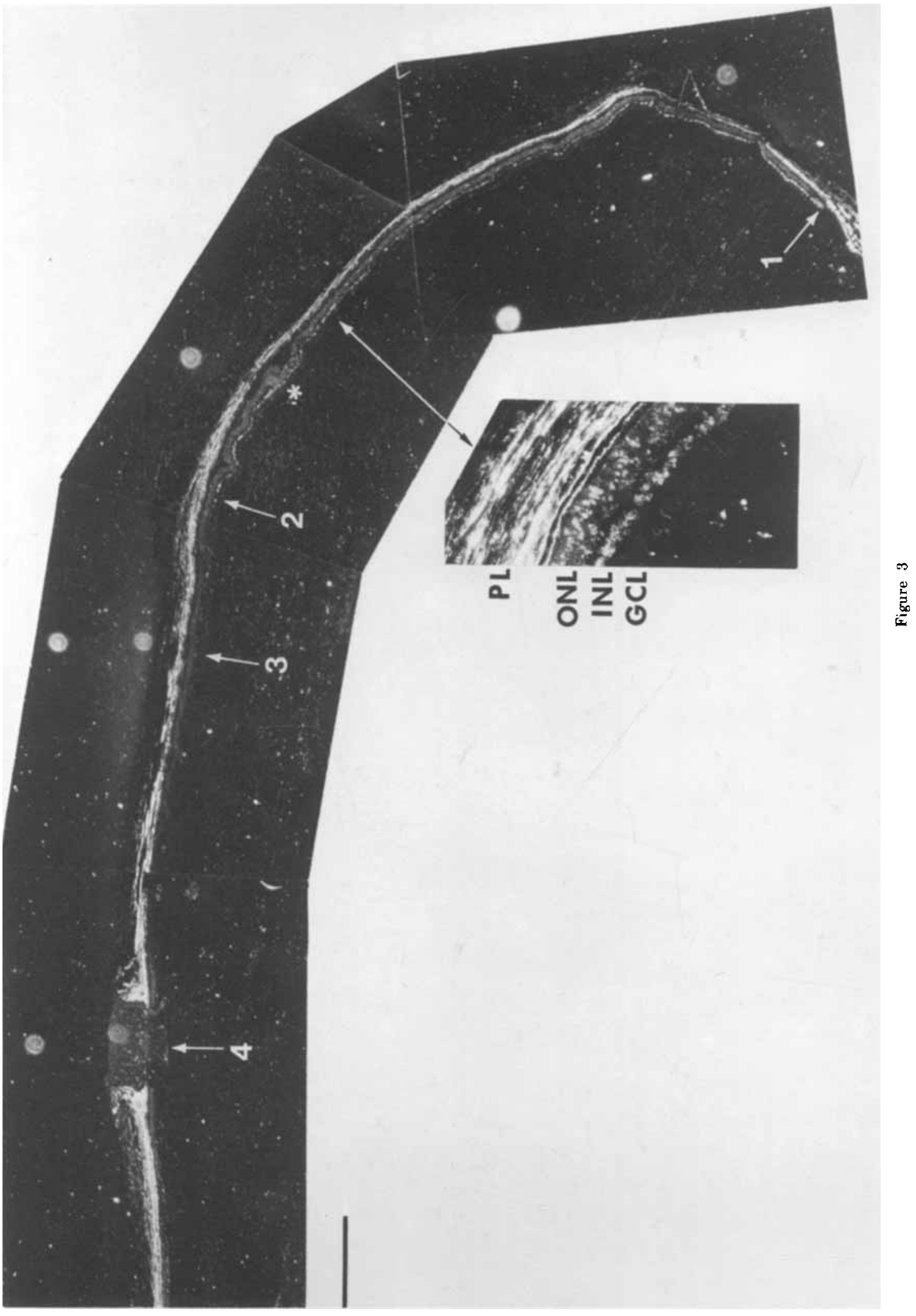


This comparison must be made with caution, however. These sections were thicker than the average diameter of the retinal cell nuclei, so some nuclei were completely contained within the section. Only those nuclei near the top of the section were close enough to the emulsion to expose it, since the effective path length of $\beta$-particles emitted by tritium is only about $1 \mu \mathrm{m}$ (Rogers, '73). All nuclei deeper than a few micrometers in the section were seen as unlabeled, even those which contained tritium $\left({ }^{3} \mathrm{H}\right)$. The observed labeling indices are, there-
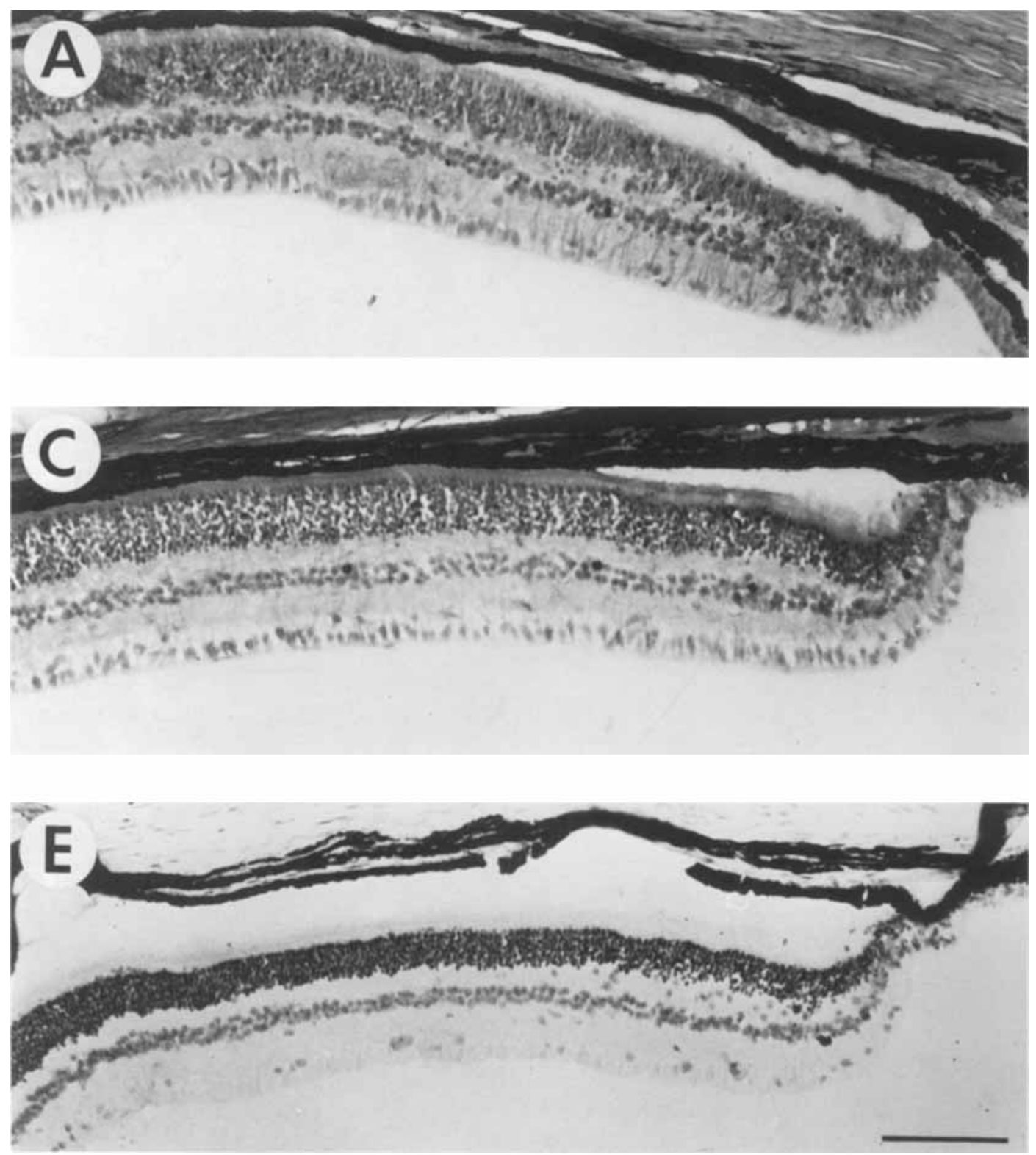

Fig. 4 Paired bright-field and dark-field radioautographs of the peripheral, dorsal retina from 14-week-old kittens, injected with ${ }^{3} \mathrm{H}$-thymidine 1 day, 10 days or 3 weeks after birth. Each pair (A, B; C, D; E, F) is the same section printed in mirror-image to facilitate comparison of the labeled regions. The dashed line on the dark-field radioautographs indicates the inner limiting membrane. 
fore, underestimates. The magnitude of this error due to "hidden" labeled nuclei is larger for the outer nuclear layer, since the nuclei there are smaller than those in the inner nu- clear layer. The lower labeling index observed in the outer compared with the inner nuclear layer is thus at least in part due to this discrepancy. To correct the labeling indices
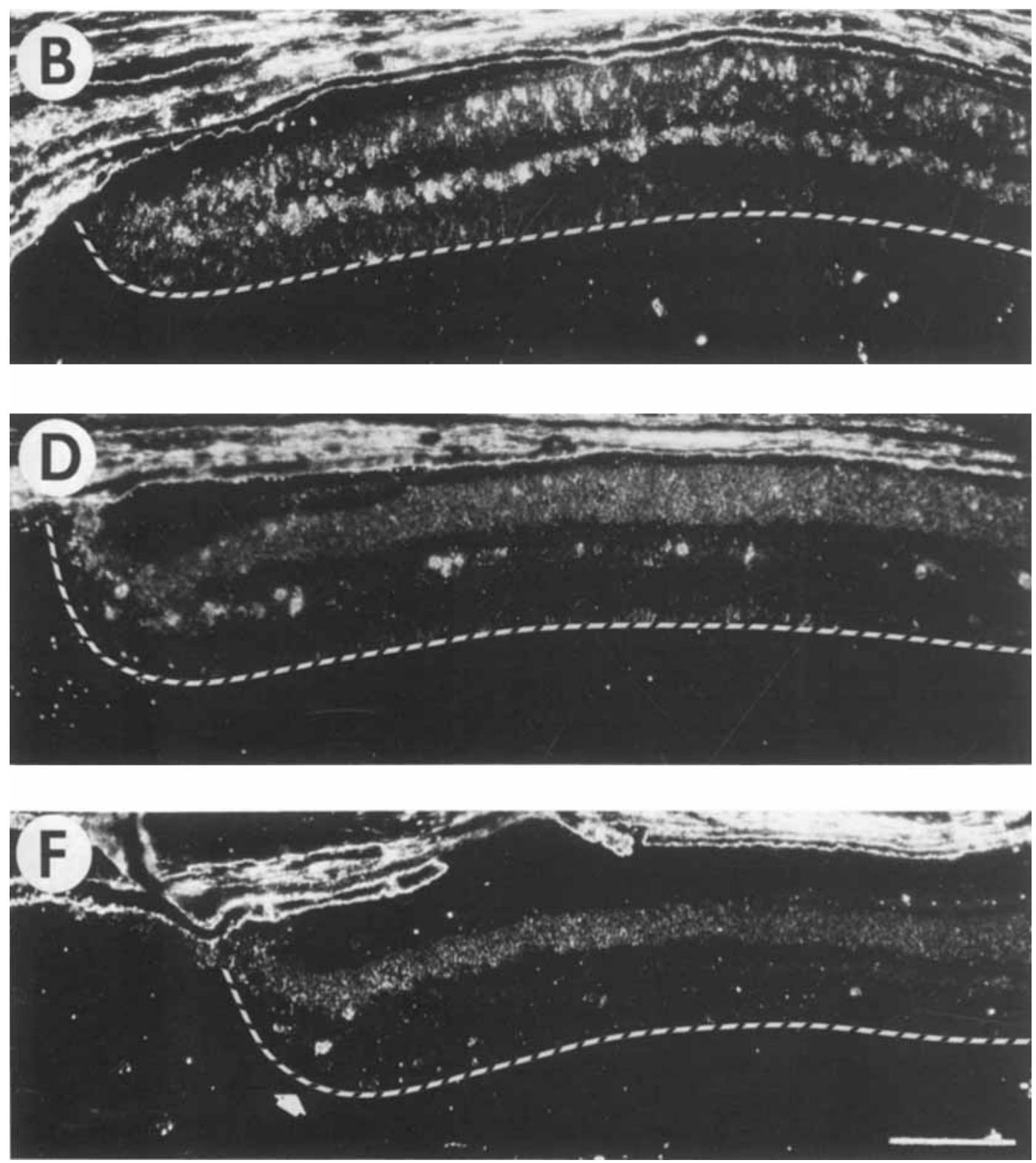

A, B Radioautographs from the retina of a kitten injected one day after birth. Many labeled nuclei are seen in the inner and outer nuclear layers but none in the ganglion cell layer.

C, D Radioautographs from the retina of a kitten injected ten days after birth. Labeled nuclei are seen in the inner nuclear layer and a few in the outer nuclear layer.

E, F Radioautographs from the retina of a kitten injected three weeks after birth. The arrow points to the only two labeled nuclei seen in this section. The scale equals 100 micrometers. 
TABLE 1

Extent of retina with labeled nuclei ${ }^{1}$

\begin{tabular}{clccc}
\hline $\begin{array}{c}\text { Age } \\
\text { (at injection) }\end{array}$ & Layer & Dorsal & Ventral & $\begin{array}{c}\text { Total retinal } \\
\text { length }{ }^{2}\end{array}$ \\
\hline \multirow{2}{*}{1 day } & INL & $11.0(9.7-11.7)$ & $10.0(8.7-10.7)$ & 25.9 \\
& ONL & $8.3(7.1-9.4)$ & $8.4(7.2-9.8)$ & 26.8 \\
10 days & INL & $7.3(5.3-9.7)$ & $5.2(3.9-6.5)$ & $1.7(1.6-1.8)$ \\
& ONL & $2.5(0.9-4.1)$ & $0.04(0.0-0.09)$ & 22.4 \\
3 weeks & INL & $0.1(0.05-0.15)$ & $0.01(0.0-0.03)$ & \\
& ONL & $0.04(0.03-0.05)$ & No labeled cells identified as neurons \\
\hline
\end{tabular}

Measurements were made on one retina at each age, which was sectioned in the dorsoventral plane through the optic disc. In the column labeled dorsal are the lengths of retina in millimeters, from the dorsal ora serrata toward the optic disc, in which labeled nuclei were found in the inner (INL) and outer (ONL) nuclear layers. In the column labeled ventral are the analogous measures from the ventral side of the game sections. Each number is the mean of measurements on five sections. Parentheses enclose the range of measurements.

${ }^{2}$ The length of the retina in hillimeters from the dorsal ora serrata through the optic disc to the ventral ora serrata, measured at the level of the INL. Since all kittens surived to 14 weeks of age, this length should be the same for all eyes; differenceg are accounted for by differential shrinkage during the histology. The first two eyes were embedded in carbowax and the third ( 3 weeks old at injection) was embedded in paraffin, in which tissue shrinkage was greater.

would require that we estimate the values of several critical parameters more accurately than we can.

When thymidine was injected into the eye at ten days after birth and the kitten allowed to survive to the same age as before (14 weeks), many fewer labeled nuclei were seen (figs. 4C,D), and they were confined to a shorter segment of the retina, again extending centrally from the ora serrata. The most central $55 \%$ of the length of the retina contained no labeled nuclei, indicating that all of the neurons in this region complete their terminal division by ten days after birth. In the inner nuclear layer, only 5-7 mm of retina adjacent to the ora serrata ( $45 \%$ of the total length of $26.8 \mathrm{~mm}$ ) contained labeled nuclei (table 1). There were many labeled nuclei in the inner nuclear layer near the peripheral margin of the retina, but more centrally only a few labeled nuclei were found, scattered among unlabeled cells. As in the animals injected at one day, labeled nuclei in the outer nuclear layer were confined to a shorter segment of the retina: only about $2 \mathrm{~mm}$ of the outer nuclear layer adjacent to the ora serrata, or $15 \%$ of its total length, contained labeled nuclei (table 1). Within the labeled region of the outer nuclear layer fewer nuclei were seen more centrally, and the proportion of labeled nuclei in this layer appeared smaller than that in the inner nuclear layer, although the cautions described above also apply here.

Radioautographs from retinas injected at three weeks after birth showed only a few labeled nuclei very near the ora serrata (figs.
$4 \mathrm{E}, \mathrm{F} ;$ table 1 ). Most of these nuclei were in the inner nuclear layer, although a few labeled nuclei were also observed in the outer nuclear layer. Retinas from kittens injected at four weeks after birth had no labeled neuronal nuclei. Thus, the last retinal neurons are formed at the peripheral margin at about three weeks after birth in the kitten.

Two comments relate to potential artifacts seen in the radioautographs. Both of the eyes injected at one day after birth showed some evidence of trauma or mechanical damage. In several places the outer nuclear layer was thrown into folds and swirls; an example is seen in figure 3 . These distortions might have affected our measurements of the length of labeled retina, although the boundary between labeled and unlabeled regions in the sections measured never fell on or near a fold, and the inner nuclear layer appeared normal everywhere. Even if the values for length of labeled retina given in table 1, particularly those for the outer nuclear layer, are not exact, we are confident that the pattern of labeling observed was real; in the animals injected at ten days, whose retinas showed no signs of damage, the same pattern of labeling was seen.

A second problem also relates to the mechanics of the injection. In some eyes the length of labeled retina was longer on the dorsal than on the ventral side (table 1), but the reverse was not seen. ${ }^{4}$ This suggests that post-

4 The only apparent exception is the length of labeled outer nuclear layer in the animal injected one day after birth: $8.3 \mathrm{~mm}$ (dorsa) and $8.4 \mathrm{~mm}$ (ventral). These values are equivalent within our ex. perimental accuracy. 
natal cell proliferation may be more extensive in the dorsal than in the ventral retina. This suggestion is further supported by a similar inequality in the extent of retina containing mitotic figures in the newborn kitten retina: the dorsal segment with mitotic figures was $9.8 \mathrm{~mm}$ long whereas the ventral segment was only $5.9 \mathrm{~mm}$ long. But because the length of retina containing mitotic figures was measured in only one animal, and because the needle was placed in the dorsal-temporal quadrant of the eye for each injection, we cannot at present discount the possibility that the observed asymmetry in labeling was due to unequal distribution of the ${ }^{3} \mathrm{H}$-thymidine precursor.

\section{DISCUSSION}

Production of neurons in the newborn kitten retina has been implied by previous reports of mitotic figures at the retina-pigmented epithelium boundary (Donovan, '66; Morrison, '77; Vogel, '78). However, neither the location of the mitotic cells along the centralto-peripheral dimension nor the fate of the dividing cells was determined in these stud. ies. In our sections of newborn kitten retina, cut parallel to the dorsoventral plane and passing through the optic disc, mitotic figures were seen across most of the extent of the retina, being absent only in the central 20\% around the disc. Recognizing that the presence and number of mitotic figures is an unreliable gauge of the level of proliferative activity of a cell population (since the mitotic phase is only a small fraction of the total cell cycle [Fujita, '62]), we used ${ }^{3} \mathrm{H}$-thymidine radioautography to describe the pattern of cell proliferation more accurately. The advantages of this technique are that dividing cells can be identified at any stage of the cell cycle, not just during mitosis, and that they are tagged with a more or less permanent label. With radioautography we have shown that postnatal neurogenesis in the kitten retina is much more extensive than previously thought: $80 \%$ of the linear extent of the retina along the dorsoventral meridian is actively producing cells one day after birth. We have traced the progeny of the proliferating cells in the radioautographs and shown that they are destined for the outer and inner nuclear layers where they differentiate into photoreceptors and other neurons (and perhaps also Müller cells), respectively. None of the new cells produced one day after birth or later become ganglion cells. Vogel (78) thought that the dividing cells in the newborn kitten retina contributed only to the outer nuclear layer since mitotic figures were found only in that layer. But the restriction of mitotic figures to the retina-pigmented epithelium boundary is expected. Mitosis of neuroepithelial germinal cells always occurs at the ventricular surface (Fujita, '62), and, in the eye, the junction between retina and pigmented epithelium is the former optic ventricle (Mann, '69). After dividing, the nuclei move away from the ventricular surface; those produced postnatally in the kitten retina go to either the outer or the inner nuclear layer.

A comparison of the distribution of mitotic figures in the newborn kitten retina with the radioautographic measurements on the animals injected at one day of age is informative: both mitotic figures and labeled nuclei were restricted to the peripheral $80 \%$ of the retinal length. Thus the fraction of retina containing mitotically active cells at birth and that containing their labeled progeny at 14 weeks is equal in both position and length. This implies that the cells produced postnatally do not migrate laterally along the retinal layers, but rather differentiate in situ. Since the ganglion cell layer is complete from disc to ora serrata at one day after birth, postnatal retinal neurogenesis serves only to "fill in" cells in the more scleral layers. Incremental addition of rings of totally new retinal tissue such as occurs in growing, postembryonic fish and amphibian retinas (Straznicky and Gaze, '71; Johns, '77), does not, therefore, happen in the kitten. Nevertheless, the kitten retina does grow postnatally: in one newborn animal the length of the retina was $19.1 \mathrm{~mm}$, but in the 14-week-old animal injected one day after birth, the retinal length was 25.9 mm-a linear increase of $26 \%$. We suggest that this growth is due to stretching ${ }^{5}$ of existing retinal tissue. Consistent with this idea of retinal stretching, Tucker ('78) has shown that the distance between optic disc and area centralis increases with the age of the kitten. From her figure 4 we calculate that between one week and four months of age the distance from disc to area centralis increases by $20 \%$. Further quantitative studies are needed to define the exact geometry of the retinal expansion. For example, does the retina stretch symmetrically throughout its length and in

\footnotetext{
"The word "stretching" is not meant to imply a mechaniem of retinal growth, but simply an end result; the retina grows larger as does a balloon when filled with air.
} 
all meridians? Studies designed to answer these and related questions are in progress (for further discussion see Rusoff, '79).

The following general conclusions can be made about the postnatal development of the kitten retina. By one day after birth, and perhaps much sooner, the ganglion cells have ceased dividing and begun differentiating across the entire extent of the retina. Many of the photoreceptor nuclei in the outer nuclear layer are also formed before birth and so are not labeled when ${ }^{3} \mathrm{H}$-thymidine is injected postnatally. The latter conclusion is supported by the relatively differentiated appearance of these nuclei across much of the outer nuclear layer at birth. The inner nuclear layer is the least mature at birth. Only in the central region of the retina do these cells have differentiated nuclei; elsewhere their nuclei remain immature or neuroblastic. In peripheral retina, many of the cells of the inner nuclear layer are born postnatally. (The order of birth of the various cells in the inner nuclear layer was not determined here, since the different types could not be distinguished in our material.) Thus, neurogenesis in the kitten retina follows the general pattern described for the rat (Denham, '67), the mouse (Sidman, '60) and the rabbit (McArdle et al., '77): (1) maturation proceeds from center to periphery, (2) ganglion cells are formed prenatally, and (3) the final stages of neurogenesis in peripheral regions of the inner and outer nuclear layers occur after birth.

The early, most active period of postnatal neurogenesis occurs while the kitten's eyes are closed, from birth until seven to ten days later. From ten days to three weeks after birth, neurons continue to be produced but only in the most peripheral regions. During this time behavioral evidence of visual function begins to appear: orienting behavior at 15 days and placing responses at 21 days (Norton, '74). The last retinal neurons are produced at about three weeks. Physiological maturation of the retina continues beyond this point, as the retinal ganglion cells gradually develop their mature response properties (Hamasaki and Flynn, '77; Rusoff and Dubin, '77). Other visual centers are also maturing at this late stage. In addition, numerous experiments have demonstrated the existence of a "critical period," during which time the visual stimulation the animal receives has a powerful influence on the continued development and/or maintenance of visual function (Hubel and Wiesel, '70; for a review of the literature see Blakemore, '74; Barlow, '75; Daniels and Pettigrew, '76). It is interesting to note that the "critical period" does not begin until about four weeks after birth, just at the time when retinal neurogenesis has ceased.

\section{ACKNOWLEDGMENTS}

We wish to thank Dr. S. S. Easter for suggesting the experiment, for providing histological facilities and for his comments on the manuscript. We also thank Dr. Leslie J. Fisher for useful discussions, Dr. Patric Clapshaw for helping with the initial injections and for providing the ${ }^{3} \mathrm{H}$-thymidine used for those injections, Ms. Janine Easter and Ms. Gertrude Callaway for helping with the histology, and Ms. DeAnn Madrid and Ms. Corrinne Mannhalter for typing the manuscript. This work was supported by National Science Foundation Grant BNS76-00506 to M. W. Dubin; P. R. Johns was supported by a National Research Service Award, NS 05518-01, from the Public Health Service.

\section{LITERATURE CITED}

Barlow, H. B. 1975 Visual experience and cortical development. Nature, 258: 199-204.

Blakemore, C. 1974 Development of functional connexions in the mammalian visual system. Br. Med. Bull., 30: 152-157.

Cragg, B. G. 1975 The development of synapses in the visual system of the cat. J. Comp. Neur., 160: 147-166.

Daniels, J. D., and J. D. Pettigrew 1976 Development of neuronal responses in the visual system of cats. In: Neu. ral and Behaviorial Specificity. Studies on the Development of Behavior and the Nervous System. G. Gottlieb, ed. Acad. Press, New York, pp. 195-232.

Denham, S. 1967 A cell proliferation study of the neural retina in the two-day rat. J. Embryol. Exp. Morph., 18: 53-66.

Donovan, A. 1966 The postnatal development of the cat retina. Exptl. Eye Res., 5: 249-254.

Fujita, S. 1962 Kinetics of cellular proliferation. Exp. Cell Res., 28: 52-60.

Hamasaki, D. I., and J. T. Flynn 1977 Physiological properties of retinal ganglion cells of three week old kittens. Vision Res., 17: 275-284.

Hinds, J. W., and P. L. Hinds 1974 Early ganglion cell differentiation in the mouse retina: An electron microscopic analysis utilizing serial sections. Devel. Biol,, 37: 381-416.

Hubel, D. H., and T. N. Wiesel 1970 The period of susceptibility to the physiological effects of unilateral eye closure in kittens. J. Physiol., 206: 419-436.

Johns, P. R. 1977 Growth of the adult goldfish eye. III. Source of the new retinal cells. J. Comp. Neur., 176: 343-358.

Kopriwa, B. M., and C. P. Leblond 1962 Improvements in the coating technique of radioautography. J. Histochem. Cytochem., 10: 269-284. 
Mann, I. 1969 The Development of the Human Eye. Grune and Stratton, New York.

McArdle, C. B., J. E. Dowling and R. H. Masland 1977 Development of outer segments and synapses in the rabbit retina. J. Comp. Neur., 175: 253-274.

Morrison, J. D. 1977 Electron microscopic studies of developing kitten retina. J. Physiol., 273: 91P-92P.

Norton, T. T. 1974 Receptive field properties of superior colliculus cells and development of visual behavior in kittens. J. Neurophysiol., 37: 674-690.

Ramóm y Cajal, S. 1960 Studies on Vertebrate Neurogenesis. Translated by L. Guth. Thomas, Springfield, Illinois, pp. 353-379.

Rogers, A. W. 1973 Techniques of Autoradiography. Elsevier Scientific Publishing Co., Amsterdam.

Rusoff, A. 1979 Development of ganglion cells in the retina of the cat. In: Developmental Neurobiology of Vision. R. D. Freeman, ed. Plenum Press.

Rusoff, A. C., and M. W. Dubin 1977 Development of receptive field properties of retinal ganglion cells in kittens. J. Neurophysiol., 40: 1188.1198.

Sidman, R. L. 1960 Histogenesis of mouse retina studied with thymidine- $\mathrm{H}^{3}$, In: The Structure of the Eye. G, K. Smelser, ed. Acad. Press, New York, pp. 487-506.

Straznicky, K., and R. M. Gaze 1971 The growth of the retina in Xenopus laevis: an autoradiographic study. J. Embryol. Exp. Morph., 26: 67-79.

Tucker, G. S. 1978 Light microscopic analysis of the kitten retina: postnatal development in the area centralis. J. Comp. Neur., 180: 489-500.

Vogel, M. 1978 Postnatal development of the cat's retina. Adv. Anat. Embryol. Cell Biol., 54: 1-66. 